\title{
Molecular Dynamics Simulations to Determine the Structure and Dynamics of Hepatitis B Virus Capsid Bound to a Novel Anti-viral Drug
}

\author{
Go Watanabe, ${ }^{a}$ Shunsuke Sato, ${ }^{a}$ Mitsuo Iwadate, ${ }^{b}$ Hideaki Umeyama, ${ }^{b}$ Michiyo Hayakawa, ${ }^{c}$ \\ Yoshiki Murakami, ${ }^{c}$ and Shigetaka Yoneda*,a \\ ${ }^{a}$ School of Science, Kitasato University; 1-15-1 Kitasato, Minami-ku, Sagamihara 252-0373, Japan: ${ }^{b}$ Department \\ of Biological Science, Chuo University; 1-13-27 Kasuga, Bunkyo-ku, Tokyo 112-8551, Japan: and ${ }^{c}$ Department of \\ Hepatology, Graduate School of Medicine, Osaka City University; 1-4-3 Asahi-cho, Abeno-ku, Osaka 545-8585, \\ Japan.
}

Received February 10, 2016; accepted June 12, 2016

Hepatitis B virus (HBV) chronically infects millions of people worldwide and is a major cause of serious liver diseases, including liver cirrhosis and liver cancer. In our previous study, in silico screening was used to isolate new anti-viral compounds predicted to bind to the HBV capsid. Four of the isolated compounds have been reported to suppress the cellular multiplication of HBV experimentally. In the present study, molecular dynamics simulations of the $\mathrm{HBV}$ capsid were performed under rotational symmetry boundary conditions, to clarify how the structure and dynamics of the capsid are affected at the atomic level by the binding of one of the isolated compounds, C13. Two simulations of the free HBV capsid, two further simulations of the capsidC13 complex, and one simulation of the capsid-AT-130 complex were performed. For statistical confidence, each set of simulations was repeated by five times, changing the simulation conditions. C13 continued to bind at the predicted binding site during the simulations, supporting the hypothesis that $\mathrm{C} 13$ is a capsid-binding compound. The structure and dynamics of the HBV capsid were greatly influenced by the binding and release of $\mathrm{C13}$, and these effects were essentially identical to those seen for AT-130, indicating that C13 likely inhibits the function of the HBV capsid.

Key words rotational symmetry boundary condition; in silico screening; hepatitis B virus; capsid

Hepatitis B virus (HBV) chronically infects millions of people worldwide and is a major cause of serious liver diseases, including liver cirrhosis and liver cancer. ${ }^{1)}$ Interferon-based inhibitors and nucleic acid analogs have been approved for the treatment of HBV-related diseases in the U.S.A. However, the interferon-based inhibitors fall short of delivering ideal clinical outcomes, and the nucleic acid analogs need to be administered over a long term. Therefore, more effective and fast acting treatments targeting $\mathrm{HBV}$ are required. In our previous study, in silico screening ${ }^{2)}$ was used to isolate 16 anti-viral candidate compounds, which are predicted to be bound to the HBV capsid. ${ }^{3)}$ The candidate compounds were tested in an in vitro $\mathrm{HBV}$ infection system. The levels of HBV DNA and HBV surface antigens were significantly reduced in the PXB cells (PhoenixBio, Hiroshima, Japan) which were treated separately with four of the previously identified compounds, namely $\mathrm{C} 9, \mathrm{C} 10, \mathrm{C} 13$, and $\mathrm{C} 16$. However, it has not been established whether the compounds actually inhibit the function of the HBV capsid at the atomic level. Thus, we performed molecular dynamics (MD) simulations of the HBV capsid to model how the structure and dynamics of the capsid are likely to be affected by the binding of $\mathrm{C} 13$, one of the most effective compounds found in the previous study. The chemical name of $\mathrm{C} 13$ is 4-(4-acetylpiperazin-1-yl)- $N$-[1-(cyclopropylamino)1-oxo-3-phenylpropan-2-yl]-3-nitrobenzamide.

The structure of HBV capsid has been reported by cryoelectron microscopy. ${ }^{4,5)}$ Later, the atomic coordinates usable for the initial coordinates of MD simulation have been determined by X-ray crystallographic analysis for the free HBV capsid (Protein Data Bank [PDB] code; 1QGT), ${ }^{6}$ for the HBV capsids bound with the HAP1 compound (PDB code; 2G34), ${ }^{7}$ and for the HBV capsids bound with the AT-130 compound (PDB code; 4G93). ${ }^{8}$ ) The resolution of X-ray diffractions for $2 \mathrm{G} 34$ was rather low at $5.5 \AA(1 \AA=0.1 \mathrm{~nm})$, compared to that of $1 \mathrm{QGT}$ at $3.3 \AA$ and of $4 \mathrm{G} 93$ at $4.2 \AA$. Furthermore, the atomic coordinates of HAP1 were not determined in this $\mathrm{X}$-ray structure of the HBV-HAP1 complex. Hence, we used 1QGT and 4G93 for the initial structures in the following MD simulations. Since AT-130 is a well-studied capsid-binding drug, a comparison of the effects of $\mathrm{C} 13$ and AT-130 on the HBV capsid structure would be important in elucidating the anti-HBV mechanism of $\mathrm{C} 13$.

The three crystal structures are icosahedrally symmetric, with a $\mathrm{T}$ number of 4 . Thus the entire capsid is composed of 60 identical protein units, and each protein unit is composed of four identical peptide chains, A, B, C, and D. Each chain has a distinctive spike composed of two long $\alpha$-helices connected by a loop. The A and B chains tend to make a stable dimer, as well as the $\mathrm{C}$ and $\mathrm{D}$ chains. The compounds, C9, $\mathrm{C} 10, \mathrm{C} 13$, and $\mathrm{C} 16$, are predicted to occupy the two putative sites that are at the base of the $\mathrm{A} / \mathrm{B}$ and $\mathrm{C} / \mathrm{D}$ dimers near one of the two 2-fold axes.

A single peptide chain of the HBV capsid protein contains around 150 amino acid residues, and the entire capsid structure is composed of around 36000 residues. MD simulations of this large protein assembly consume an exceptionally large amount of computer time, even when using current, state-of-the-art high-speed computers. In order to accelerate simulations of symmetric assemblies, the rotational symmetry boundary condition (RSBC) approach has been developed.9) Because RSBC restricts calculations for the entire symmetric assembly to a single asymmetric unit, the calculations are 
Table 1. Initial Structures of Simulations

\begin{tabular}{lcc}
\hline \hline Simulation & $\begin{array}{c}\text { Initial structure of } \\
\text { capsid }\end{array}$ & Ligand \\
\hline F1qgt & 1QGT & Free \\
F4g93 & $4 \mathrm{G} 93$ & Free (AT-130 was deleted) \\
B4g93 & $4 \mathrm{G} 93$ & AT-130 \\
B4g93C13 & $4 \mathrm{G} 93$ & $\mathrm{C} 13$ (AT-130 was replaced with C13) \\
B1qgtC13 & 1QGT & C13 (C13 was added) \\
\hline
\end{tabular}

greatly accelerated. RSBC has been applied to the capsids of poliovirus, rhinovirus, and foot-and-mouth disease virus, in addition to the crystal of glycogen phosphorylase $\left(P 4_{3} 2_{1} 2\right.$ space group), for pharmaceutical and biological interests. ${ }^{10-13)}$

Simulations were accelerated in RSBC, but the conformational change of this essentially large molecular assembly is believed to have an extremely long time-scale. Therefore, we compared the rate of the structural change in the capsid caused by binding with the anti-viral compounds within $500 \mathrm{ps}$. We performed five types of MD simulations on the HBV capsid under RSBC, as shown in Table 1. The simulation we named 'Flqgt,' was performed using the X-ray crystal structure of the free capsid (1QGT) as the initial model. In simulation 'B1qgtC13,' two C13 molecules were modeled into the binding sites of 1QGT. Simulation 'B4g93' was performed based on the X-ray crystal structure of the capsid-AT-130 complex, (4G93). Simulations 'F4g93' and 'B4g93C13' were performed based on the X-ray crystal structure, 4G93, as the initial model; AT-130 was deleted in 'F4g93' and AT-130 was replaced with $\mathrm{C} 13$ in 'B4g93C13.' For statistical confidence, each simulation type was repeated by five times by changing simulation conditions.

\section{Experimental}

MD Simulation The program APRICOT was used for the present MD simulations. ${ }^{9,14)}$ The AMBER 'ff12SB' energy parameters $^{15)}$ and TIP3P potentials ${ }^{16)}$ were used to model the protein and water, respectively. The atomic charges on $\mathrm{C} 13$ and AT-130 were calculated using the restrained electrostatic potential (RESP) method, ${ }^{17)}$ based on quantum chemical calculations using the B3LYP/6-31G* basis set in GAUSSIAN09. ${ }^{18)}$ Interactions from the neighboring asymmetric units were included using the protomer number equations.9) The inner and outer radii of the capsid from the center of icosahedral symmetry were 126.3 and $174.6 \AA$, respectively. The region radially enclosed between 111 and $192 \AA$ was filled with water
Table 2. The Number of Atoms for MD Simulations

\begin{tabular}{lccc}
\hline \hline Simulation & Protein & Ligand & Water \\
\hline F1qgt & 9070 & - & 25293 \\
F4g93 & 8911 & - & 24285 \\
B4g93 & 8911 & 110 & 24249 \\
B4g93C13 & 8911 & 128 & 24255 \\
B1qgtC13 & 9070 & 128 & 25290 \\
\hline
\end{tabular}

molecules, as shown in Fig. 1. The water region was large enough to encompass the larger movements of the protein including the outer spikes of the capsid. A half-harmonic potential of $1 \mathrm{kcal} /\left(\mathrm{mol} \cdot \AA^{2}\right)$ was added to the water atoms in order to prevent the escape of the atoms through the inner or outer surface of the water region. The structure of the genome in the core for the HBV capsid has not been determined by $\mathrm{X}$-ray analysis, as is true of almost all other virus capsids. A consequence of this is that the HBV capsid model tends to shrink during MD simulations by the lack of stabilizing force from the core. Thus, we added weak positional constraints of $0.1 \mathrm{kcal} /\left(\mathrm{mol} \cdot \AA^{2}\right)$ to about $30 \mathrm{C} \alpha$ atoms with low experimental B-factors, as in the previous simulations. ${ }^{12,13)}$ The number of atoms of protein, ligand, and water molecules included in the present MD simulations are listed in Table 2. A cut-off of $18 \AA$ was used for the non-bonded interactions. After energy minimizations, MD simulations for equilibration of water were performed at temperature of $300 \mathrm{~K}$ with the positional constraints for the non-water atoms. The force constant of the constraints was gradually decreased to the force constant of $5 \mathrm{kcal} /\left(\mathrm{mol} \cdot \AA^{2}\right)$ for all the non-hydrogen atoms in non-water residues. The constraint $\mathrm{MD}$ simulations were continued until $254 \mathrm{ps}$; atomic coordinates and velocities were saved at 1-ps intervals during the final 4 ps of the simulation. Data at these five points were used for the initial states for the MD simulations with length of $500 \mathrm{ps}$. Thus, five MD simulations were calculated for each initial model type.

\section{Results and Discussion}

Table 3 provides the root mean square deviation (RMSD) values of $\mathrm{C} \alpha$ atoms in the simulated capsid protein obtained by MD simulations. The RMSD values for the protein unit composed of the 4 chains, were all approximately $4 \AA$ in simulations F1qgt and B4g93, which started from the X-ray derived structural models of the free capsid and of the capsid-AT-130 complex, respectively. The RMSD after least squares fit (LS fit) represents the pure conformational changes with the rigid

Table 3. The Average RMSD of the C $\alpha$ Atoms $^{a, b, c)}$

\begin{tabular}{|c|c|c|c|c|c|}
\hline & F1qgt & F4g93 & B4g93 & B4g93C13 & B1qgtC13 \\
\hline Protein Unit & $2.7 \pm 0.6(3.5 \pm 0.5)$ & $3.4 \pm 0.5(4.0 \pm 0.5)$ & $3.0 \pm 0.6(3.8 \pm 0.5)$ & $3.0 \pm 0.5(3.7 \pm 0.5)$ & $2.9 \pm 0.4(3.8 \pm 0.4)$ \\
\hline A Chain & $2.0 \pm 0.3(2.9 \pm 0.3)$ & $2.6 \pm 0.4(3.7 \pm 0.6)$ & $2.4 \pm 0.6(3.7 \pm 1.0)$ & $2.5 \pm 0.3(3.6 \pm 0.4)$ & $1.8 \pm 0.3(4.1 \pm 1.7)$ \\
\hline B Chain & $1.6 \pm 0.1(3.6 \pm 0.6)$ & $2.0 \pm 0.2(3.3 \pm 0.5)$ & $1.9 \pm 0.3(3.5 \pm 0.3)$ & $2.0 \pm 0.4(3.0 \pm 0.6)$ & $2.0 \pm 0.3(3.4 \pm 0.6)$ \\
\hline C Chain & $1.9 \pm 0.2(3.8 \pm 0.9)$ & $3.2 \pm 0.9(4.0 \pm 1.1)$ & $2.6 \pm 0.4(3.6 \pm 0.3)$ & $2.8 \pm 0.5(3.8 \pm 0.8)$ & $2.6 \pm 0.5(3.9 \pm 0.5)$ \\
\hline D Chain & $2.1 \pm 0.4(3.5 \pm 0.7)$ & $2.9 \pm 0.3(4.8 \pm 1.0)$ & $2.7 \pm 0.8(4.2 \pm 0.9)$ & $2.5 \pm 0.2(4.3 \pm 0.8)$ & $2.0 \pm 0.4(3.5 \pm 0.4)$ \\
\hline A/B Dimer & $2.0 \pm 0.2(3.3 \pm 0.4)$ & $2.4 \pm 0.2(3.5 \pm 0.5)$ & $2.4 \pm 0.5(3.7 \pm 0.5)$ & $2.4 \pm 0.2(3.3 \pm 0.5)$ & $2.1 \pm 0.2(3.8 \pm 1.1)$ \\
\hline C/D Dimer & $2.3 \pm 0.3(3.6 \pm 0.8)$ & $3.4 \pm 0.6(4.4 \pm 1.0)$ & $2.8 \pm 0.6(3.9 \pm 0.5)$ & $2.8 \pm 0.4(4.1 \pm 0.7)$ & $2.5 \pm 0.5(3.7 \pm 0.4)$ \\
\hline
\end{tabular}

a) The RMSD (in $\AA$ ) between the initial structure and the averaged structure over the final $50 \mathrm{ps}$ of each simulation after LS fit is calculated; the mean value of five simulations for each system is listed above. $b$ ) The values after " \pm " represent standard deviations of the RMSDs of five simulations. $c$ ) The RMSD before LS fit is mentioned within the parentheses. 

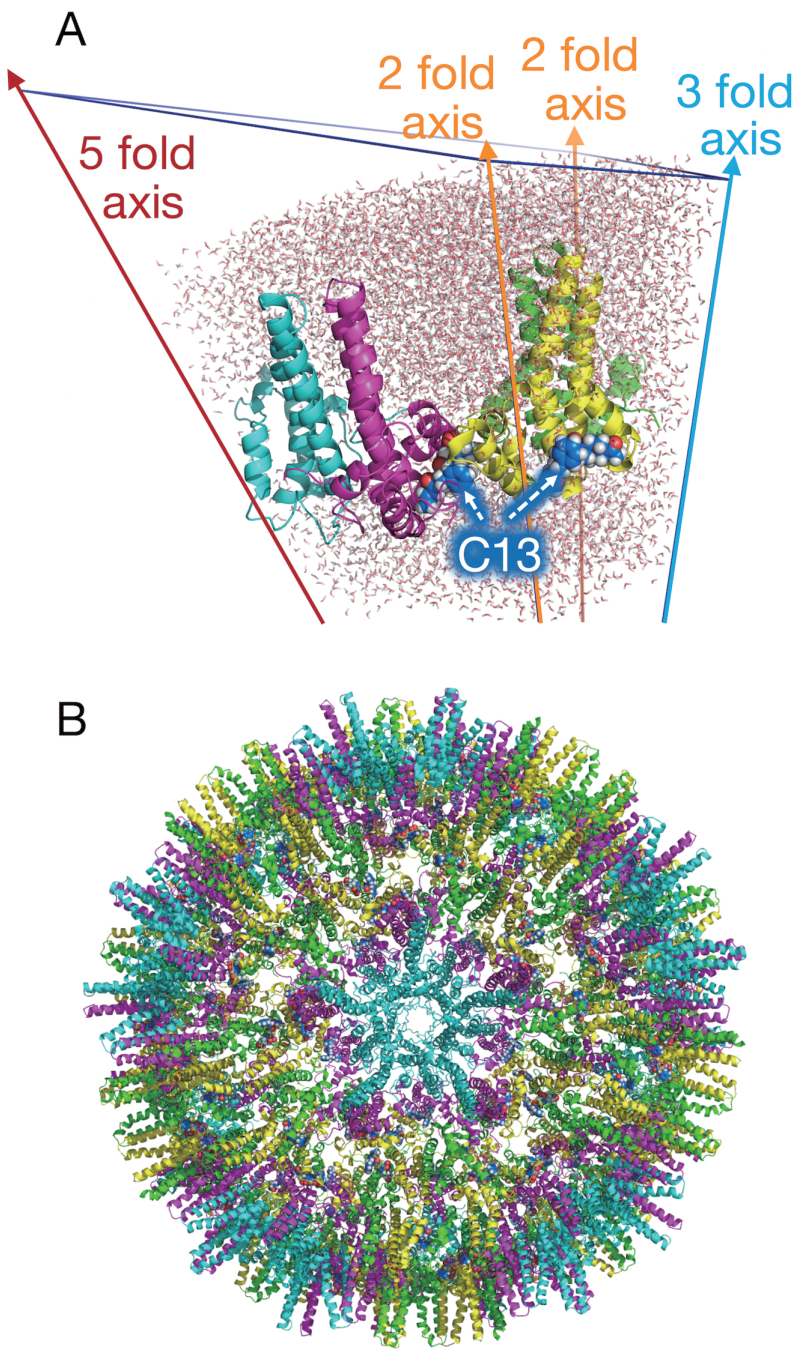

Fig. 1. Snapshot of a Representative Simulations of B4g93C13 at $500 \mathrm{ps}$

(A) The quadrangular pyramid depicts the asymmetric unit used for RSBC calculations. The top of the panel is the outer surface of the capsid, and the bottom part is the center of the entire capsid. The capsid protein is displayed as a cartoon model. The $\mathrm{C} 13$ molecules have been modeled as spheres using CPK colors. Chains A, B, C, D, and water molecules are in cyan, magenta, yellow, green, and red, respectively. (B) The entire capsid structure. Water molecules are not shown. Images were displayed using PyMOL (http://www.pymol.org).
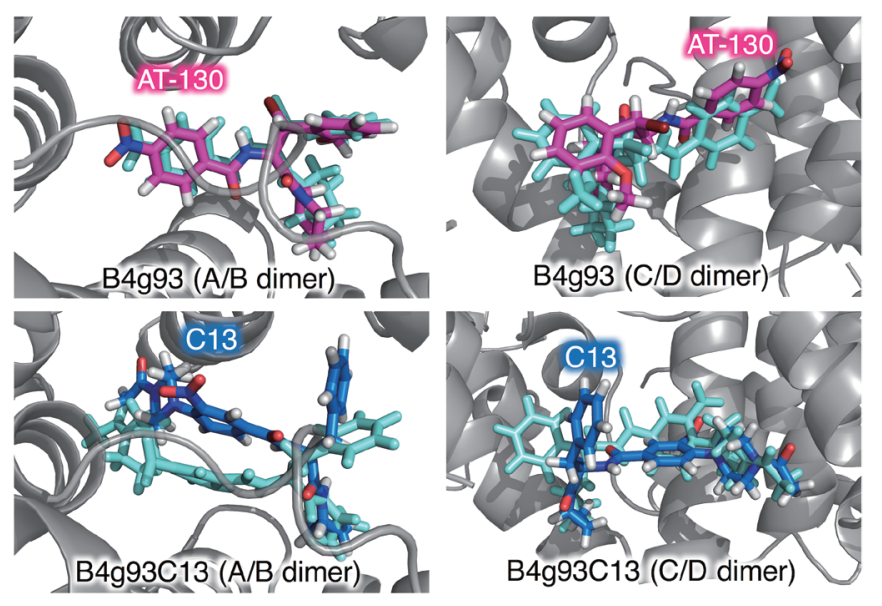

Fig. 2. Snapshot of the Two Drug-Binding Sites in the HBV Capsid

In the binding sites from one of the five simulations of B4g93, the AT-130 structures from the X-ray crystal structure, 4G93, in cyan are superimposed on those at $500 \mathrm{ps}$. The $\mathrm{C} 13$ structure in the previous in silico screening study are superimposed in cyan on those at $500 \mathrm{ps}$ in the binding sites from the five simulations of B4g93C13. Images were displayed using PyMOL.
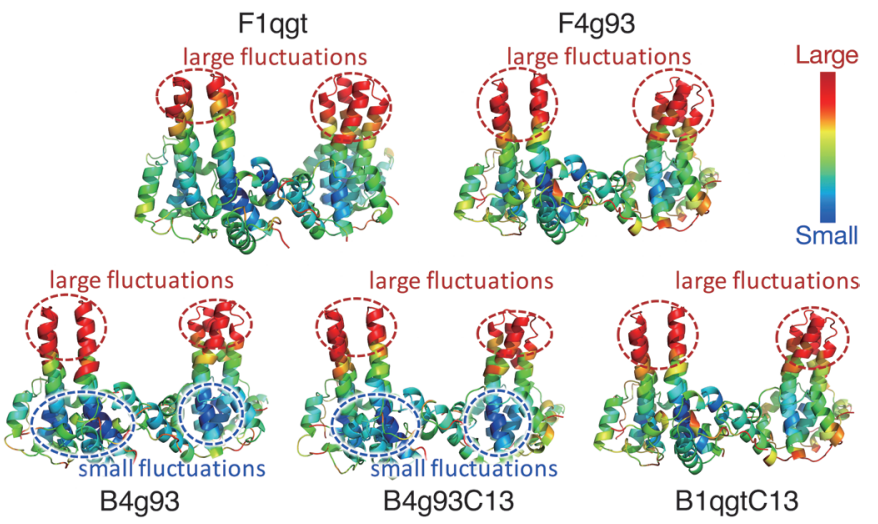

Fig. 3. The Average B-Factors Calculated from Atomic Fluctuations in the Simulations

Average structures of five simulations of HBV capsid protein units at $500 \mathrm{ps}$ from each simulation set are displayed in the same orientation as in Fig. 1. The average B-factors are depicted with color gradation. Atoms with the average Bfactors of $60 \AA^{2}$ and over are in red, and those with the average B-factors smaller than $10 \AA^{2}$ are in blue. Images were displayed using PyMOL.

translations or rotations excluded. Thus, the difference of the RMSD, before and after LS fit, represents rigid translations and rotations. In the free capsid simulation of Flqgt, the conformational change of each chain was small, below $2.1 \AA$; the change of the protein unit was $2.7 \AA$ with a standard deviation (S.D.) of $0.6 \AA$. The large part of the structural change in the protein units, was accounted for by translation and rotation of the chains, as has been reported previously. ${ }^{7.8)}$ The $\mathrm{A} / \mathrm{B}$ and $\mathrm{C} / \mathrm{D}$ dimers were also fairly rigid. The rigidity of the chains and the dimers was observed in all other simulations. The RMSD value of each chain was greater, up to $3.2 \AA$, and that of the protein unit was $3.4 \AA$ with a S.D. of $0.5 \AA$ in simulation F4g93. Considering the S.D.s of the RMSD of the protein unit, RMSD value of Flqgt was smaller than that of F4g93 with a probability of approximately $80 \%$. Here, the structural change in the HBV capsid was enhanced by the release of AT-130. The RMSD values of the protein units were equal for $\mathrm{B} 4 \mathrm{~g} 93$ and $\mathrm{B} 4 \mathrm{~g} 93 \mathrm{C} 13$ (3.0 $\AA$ ). We infer from this that the structural influence of replacing AT-130 with $\mathrm{C} 13$ in the capsid-AT-130 complex is small. The RMSD value of $2.9 \AA$ for the protein units in simulation BlqgtC13, is larger than that in Flqgt. Thus, within statistical precision of the simulations, the capsid structure in our simulation was influenced by the binding of C13 molecules onto the free capsid.

In order to analyze how AT-130 and $\mathrm{C} 13$ are bound at the active site, snapshots of the binding site structures at $500 \mathrm{ps}$ in simulations B4g93 and B4g93C13 are shown in Fig. 2. Although AT-130 and C13 fluctuated due to thermal energy, as shown in the animations made from the simulation trajectories (movies of Supplementary Materials), AT-130 and C13 continued to bind steadily at these sites during the simulations. If a molecule binds weakly with a protein, it is usual for that molecule to diffuse away during a simulation lasting $500 \mathrm{ps}$. Therefore, it is thought that $\mathrm{C} 13$ is bound well to the binding site of AT-130.

Experimental B-factors are difficult to determine precisely, since they depend on data quality, the mobility of the atoms in the crystal structure leading to uncertainty during modeling, and they can also be artificially inflated due to poor modeling during crystallographic refinement. To investigate the influ- 
ence of binding on atomic fluctuations of the protein, we calculated simulated B-factors from the final 100-ps trajectory of each MD simulation and average B-factors of five simulations, as follows ${ }^{19)}$ :

$$
B=\frac{8}{3} \pi^{2}\left\langle|\Delta \boldsymbol{r}|^{2}\right\rangle
$$

where $\left\langle|\Delta r|^{2}\right\rangle$ is the mean square atomic fluctuation relative to the average position. When the average B-factors from simulation B4g93 were compared with those from the crystal structure, 4G93 (Fig. S1 of Supplementary Materials), the relation determined using the linear regression was $y=0.36 x+2.1$, where $y$ is the average B-factor for an atom from the simulation, and $x$ is the B-factor for an atom from the crystal structure. The correlation coefficient was 0.67 . When the average B-factors from simulation F1qgt, were compared with those from the crystal structure, 1QGT, the regression equation used was $y=0.74 x+7.2$. The correlation coefficient was 0.84 . Considering these significant limitations to comparisons, the average B-factors correlated well with the B-factors reported in the models of the experimentally derived crystal structures. Figure 3 shows the distribution of positional fluctuations across the atoms in the protein models. During simulation B4g93, the tips of the spikes fluctuated greatly. In contrast, the fluctuations at the bases of the spikes were small, in agreement with reported in the previous crystallographic studies. ${ }^{6-8)}$ When AT-130 was replaced with C13, this distribution did not change and the fluctuations at the bases of the spikes remained small, as shown in Fig. 3 (B4g93C13). However, when the compound was omitted from simulation, the fluctuation greatly increased at the bases of the spikes (F4g93). In the simulation of the free capsid, Flqgt, the atomic fluctuations were small with its large fluctuations restricted to the tips of the spikes. When molecules of $\mathrm{C} 13$ were put at both binding sites during the simulation of BlqgtC13, fluctuations slightly increased especially at the base of the spikes. Therefore, the binding and release of $\mathrm{C} 13$ and AT-130 are predicted to affect the dynamic fluctuations in the structure of the HBV capsid.

A previous study ${ }^{3)}$ has experimentally demonstrated that certain compounds developed from an in silico screening, including $\mathrm{C} 13$, suppress cellular multiplication of HBV. However, no experimental evidence has been provided yet which tests the hypothesis that the compounds are actually bound to the capsid proteins, inhibiting their function at the atomic level. In the present study, a model of $\mathrm{C} 13$ was seen to remain bound at the predicted binding site of the capsid protein during MD simulations lasting 500 ps. Furthermore, an essentially identical set of structural and dynamical changes were seen in the simulated HBV capsid, when C13 and AT-130 were bound. We contend that our results predict that $\mathrm{C} 13$ inhibits at the function of the HBV capsid, in a nearly identical manner on the atomic level as AT-130. The determination of an X-ray crystal structure of the complex between the HBV capsid protein and $\mathrm{C} 13$ is expected to present detailed experimental evidence clarifying the molecular interaction, and to test the predictions that we present here.

Acknowledgments The computations were performed using Research Center for Computational Science, Okazaki,
Japan. M.H. and Y.M. were supported by KOSEI-KAKENHI, (H25-B sou-Kan-en-general-018).

Conflict of Interest The authors declare no conflict of interest.

Supplementary Materials The online version of this article contains supplementary materials.

\section{References}

1) Yang H. I., Lu S. N., Liaw Y. F., You S. L., Sun C. A., Wang L. Y., Hsiao C. K., Chen P. J., Chen D. S., Chen C. J., Taiwan Community-Based Cancer Screening Project Group, N. Engl. J. Med., 347, 168-174 (2002)

2) Murakami Y., Hayakawa M., Yano Y., Tanahashi T., Enomoto M., Tamori A., Kawada N., Iwadate M., Umeyama H., Biochem. Biophys. Res. Commun., 456, 20-28 (2015).

3) Hayakawa M., Umeyama H., Iwadate M., Tanahashi T., Yano Y., Enomoto M., Tamori A., Kawada N., Murakami Y., Biochem. Biophys. Res. Commun., 463, 1165-1175 (2015).

4) Böttcher B., Wynne S. A., Crowther R. A., Nature (London), $\mathbf{3 8 6}$, 88-91 (1997)

5) Conway J. F., Cheng N., Zlotnick A., Wingfield P. T., Stahl S. J., Steven A. C., Nature (London), 386, 91-94 (1997).

6) Wynne S. A., Crowther R. A., Leslie A. G. W., Mol. Cell, 3, 771780 (1999).

7) Bourne C. R., Finn M. G., Zlotnick A., J. Virol., 80, 11055-11061 (2006).

8) Katen S. P., Tan Z., Chirapu S. R., Finn M. G., Zlotnick A., Structure, 21, 1406-1416 (2013).

9) Yoneda S., Kitazawa M., Umeyama H., J. Comput. Chem., 17, 191-203 (1996).

10) Yoneda S., J. Mol. Graph. Model., 15, 233-237, 260 (1997).

11) Yoneda T., Yoneda S., Takayama N., Kitazawa M., Umeyama H., $J$. Mol. Graph. Model., 17, 114-119, 137-142 (1999).

12) Yoneda S., Yoneda T., Kurihara Y., Umeyama H., J. Mol. Graph. Model., 21, 19-27 (2002)

13) Azuma H., Yoneda S., J. Mol. Graph., 28, 278-286 (2009).

14) Yoneda S., Umeyama H., J. Chem. Phys., 97, 6730-6736 (1992).

15) Zgarbová M., Otypeka M., Śponer J., Mládek A., Banáš P., Cheatham T. E. III, Jurečka P., J. Chem. Theory Comput., 7, 2886-2902 (2011).

16) Jorgensen W. L., Chandrasekhar J., Madura J. D. R., Impey R. W., Klein M. L., J. Chem. Phys., 79, 926-935 (1983).

17) Bayly C. I., Cieplak P., Cornell W., Kollman P. A., J. Phys. Chem., 97, 10269-10280 (1993).

18) Frisch M. J., Trucks G. W., Schlegel H. B., Scuseria G. E., Robb M. A., Cheeseman J. R., Scalmani G., Barone V., Mennucci B., Petersson G. A., Nakatsuji H., Caricato M., Li X., Hratchian H. P., Izmaylov A. F., Bloino J., Zheng G., Sonnenberg J. L., Hada M., Ehara M., Toyota K., Fukuda R., Hasegawa J., Ishida M., Nakajima T., Honda Y., Kitao O., Nakai H., Vreven T., Montogomery J. A. Jr., Peralta J. E., Ogliraro F., Bearpark M., Heyd J. J., Brothers E., Kudin K. N., Staroverov V. N., Kobayashi R., Normand J., Raghavachari K., Rendell A., Burant J. C., Iyengar S. S., Tomasi J., Cossi M., Rega N., Millam J. M., Klene M., Knox J. E., Cross J. B., Bakken V., Adamo C., Jaramillo J., Gomperts R., Stratmann R. E., Yazyev O., Austin A. J., Cammi R., Pomelli C., Ochterski J. W., Martin R. L., Morokuma K., Zakrzewski V. G., Voth G. A., Salvador P., Dannenberg J. J., Dapprich S., Daniels A. D., Farkas Ö., Foresman J. B., Ortiz J. V., Cioslowski J., Fox D. J., Gaussian 09 (Revision D.01), Gaussian Inc., Wallingford CT, 2013.

19) McCammon J. A., Harvey S. C., "Dynamics of Proteins and Nucleic Acids," Cambridge University Press, Cambridge, 1987. 